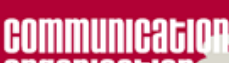
Oryanllatín.
Communication et organisation

Revue scientifique francophone en Communication organisationnelle

52 | 2017

Communauté, sociabilité et bien commun: approche internationale

\title{
Les réseaux sociaux numériques dans l'histoire du capitalisme et des transformations de la grande entreprise
}

Camille Imhoff

\section{CpenEdition}

Journals

Édition électronique

URL : https://journals.openedition.org/communicationorganisation/5734

DOI : 10.4000/communicationorganisation.5734

ISBN : 979-10-300-0257-7

ISSN : 1775-3546

Éditeur

Presses universitaires de Bordeaux

\section{Édition imprimée}

Date de publication : 1 décembre 2017

Pagination : 145-164

ISBN : 979-10-300-0163-1

ISSN : $1168-5549$

Référence électronique

Camille Imhoff, « Les réseaux sociaux numériques dans l'histoire du capitalisme et des transformations de la grande entreprise », Communication et organisation [En ligne], 52 | 2017, mis en ligne le 01 décembre 2020, consulté le 02 janvier 2022. URL : http://journals.openedition.org/ communicationorganisation/5734 ; DOI : https://doi.org/10.4000/communicationorganisation.5734 


\title{
Les réseaux sociaux numériques dans l'histoire du capitalisme et des transformations de la grande entreprise
}

\author{
Comille Imhoff ${ }^{1}$
}

\section{Introduction}

Après l'explosion des réseaux sociaux grand public, les grandes entreprises sont elles-mêmes impactées par les réseaux sociaux numériques internes depuis la fin des années 2000. Dans la communication d'entreprise et la rhétorique managériale, il s'agirait d'un décloisonnement et d'une remise en cause forcée des structures hiérarchiques. En effet, avec les réseaux sociaux d'entreprise, les grandes entreprises n'adoptent pas seulement un ensemble de technologies, mais l'idéologie ou l'esprit qui les accompagne, celui d'une ouverture de l'information, d'une plus grande horizontalité des relations, d'une répartition du pouvoir. L'expression " révolution numérique » est devenue courante, d'abord dans la société et de plus en plus dans l'entreprise pour désigner une rupture avec le fonctionnement traditionnel des organisations. Assiste- $\mathrm{t}$-on pour autant, comme nombre le prétendent ou $\mathrm{y}$ aspirent, à une forme de démocratisation des organisations qui permettrait de briser les barrières hiérarchiques et de développer une intelligence collective comme bien commun, en rupture avec le système capitaliste, ou bien s'agit-il d'une nouvelle phase du capitalisme, une nouvelle façon d'accumuler du capital, un capital social et un capital de savoir corrélés? Dans le contexte des organisations, l'usage des technologies relationnelles présente un ensemble de contradictions : entre réticence et adhésion; entre méfiance généralisée à l'égard de l'usage de plateformes numériques jugées non sérieuses, non professionnelles et pourtant preuve de son engagement ; entre nouveaux dispositifs de contrôle (les traces laissées dans loutil, les normes collectives, la surveillance interindividuelle, l'autodiscipline) et mise en visibilité de son profil, de ses activités et de son carnet d'adresses ; entre inscription

1 Camille Imhoff est doctorante contractuelle en codirection au laboratoire DICEN-IDF au Cnam et au laboratoire SOPHIAPOL à l'Université Paris-Nanterre en Sciences de l'information et de la communication ; Philosophie ; 8 rue de Prague, Escalier A. 75012 Paris ; camille.imhoff@wanadoo.fr 
idéalisée dans un réseau relationnel, selon une logique de don et contre-don désintéressée, visant le développement d'une intelligence collective comme bien commun et logique utilitariste dans la constitution de son réseau comme ressource individuelle, comme capital social c'est-à-dire comme l'ensemble des relations qu'une personne peut accumuler comme source de valeur (Bourdieu 1985). Plutôt qu'une "révolution " brutale et radicale, il semble que les technologies numériques s'inscrivent dans l'histoire des transformations du capitalisme et de ses critiques (Barbrook, Cameron 1996 ; Harvey 2005 ; Turner 2006). En effet, dans la continuité du courant critique, il s'agit d'analyser le capitalisme non pas comme une époque historique unifiée, mais davantage comme un phénomène ayant sa propre historicité et se réadaptant sans cesse pour survivre (Boltanski et Chiapello 1999). Le capitalisme présente des formes suffisamment distinctes pour pouvoir être identifiées : capitalisme marchand, industriel, financier, cognitif. Le capitalisme cognitif se caractérise par l'exploitation d'un travail immatériel (Hardt et Negri 2000; Moulier-Boutang 2007; Vercellone 2014). Le rapport capital/travail et l'entreprise capitaliste connaissent autant de formes et de réadaptations successives (Negri et Vercellone 2008) puisque l'efficacité passe du contrôle de la force de travail au développement des capacités d'apprentissage et d'innovation de la ressource humaine. Le paradigme du réseau s'impose pour décrire la forme contemporaine du capitalisme dans un contexte économique de mondialisation et d'émergence d'un marché de produits à haute valeur ajoutée. L'organisation devient un réseau d'acteurs mobiles et flexibles, dont la force réside dans l'adaptation constante au changement, au regard de la forme « organisation » et de la forme «marché » qui l'ont précédée (Powell 1990). Les technologies numériques d'information et de communication s'inscrivent au paroxysme de la société en réseau. Mais les tout derniers développements du web social avec les réseaux sociaux en ligne représentent-ils une simple accélération de la mise en réseau ou contribuent-ils à définir une nouvelle étape dans l'histoire du capitalisme ? En effet, on assiste à un déploiement viral des technologies numériques qui permettent de faire un bond en avant dans la prolifération du réseau. Les technologies de réseau social rendent possible la construction de son identité relationnelle, la visibilité de son réseau de relations comme preuve de confiance et donc l'accumulation d'un capital social à un niveau jamais imaginé jusqu'alors. Mais les réseaux sociaux numériques ne permettent pas seulement d'accroître la quantité possible de relations à accumuler, ils contribuent également à diversifier la mise en réseau quant au type de personnes connectées, au type de relation et au type d'accès. Peut-être pouvons-nous voir, dans le capitalisme cognitif qui se dessine un ensemble de transformations bien plus essentielles dans la construction des individus, des relations interindividuelles et des rapports de pouvoir au sein d'une société. La difficulté d'analyse du capitalisme contemporain est due à sa nature complexe. Cette complexité est due notamment au décloisonnement du capitalisme : 
géographique (à travers la mondialisation), historique (constitué de différentes phases) et social (se diffusant dans la diversité des univers sociaux) (Haber 2013). Tentons cependant d'en caractériser les mécanismes d'exploitation et d'aliénation spécifiques puisque « les recompositions du capitalisme appellent une ré-adéquation des termes de la critique aux nouvelles formes d'exploitation et d'aliénation que développe le capital » (Sarrouy 2013).

Dès lors, quelles sont les ruptures et les continuités dans l'histoire longue du capitalisme et de la grande entreprise qui permettent de comprendre l'opportunité et l'apparente nécessité des technologies numériques de réseau social dans les entreprises aujourd'hui ?

Nous reviendrons sur les transformations du capitalisme et plus particulièrement sur les caractéristiques propres au néocapitalisme pour interroger ensuite l'impact des technologies relationnelles numériques sur le néocapitalisme.

\section{Le néocapitalisme et les transformations de la grande entreprise}

\section{Le capitalisme dans l'histoire ou l'histoire du capitalisme?}

Le capitalisme désigne un système économique caractérisé par la propriété privée des moyens de production, la marchandisation et la recherche du profit. Marx analyse et critique ce mode de production au livre I du Capital (même s'il n'emploie pas le terme "capitalisme ») à partir de trois notions : l'exploitation, l'aliénation et l'idéologie. En effet, le capitalisme prend la forme paradigmatique du salariat, contrat ou lien de subordination juridique par lequel le travailleur vend sa force de travail contre la rémunération de son employeur qui possède les moyens de production. Mais la valeur créée par le travail n'est pas rétribuée à la mesure de sa contribution, ce qui crée une survaleur ou plus-value pour le détenteur du capital et permet une accumulation illimitée. La production capitaliste se définit par la recherche continue de l'amélioration de la productivité, c'est-à-dire le rapport optimal entre la production de biens et les ressources mises en œuvre. Le mode de production capitaliste se comprend donc essentiellement par une théorie de l'exploitation. Outre l'exploitation du travailleur, le capitalisme se définit par des caractéristiques plus empathiques que les Manuscrits de 1844 étudient sous le nom d'aliénation. L'aliénation désigne non seulement la dépossession du produit de son travail, mais aussi la dépossession de sa puissance vitale, de son rapport à soi et aux autres (Haber 2008 ; Renault 2006). Enfin, l'idéologie (ou les idées dominantes conditionnées par la position de classe et les intérêts des acteurs) justifie et maintient la domination de classe dans le système capitaliste.

Dans la pensée de Marx, le capitalisme est défini comme un mode de production relativement homogène, celui du capitalisme industriel qui fait 
suite à la révolution industrielle. Il constitue une étape définie dans la logique historique qui mène du communisme primitif au socialisme, en passant par l'esclavagisme et le féodalisme, comme analysé dans la Contribution à la critique de l'économie politique. Mais, pouvons-nous parler du capitalisme comme d'une période historique délimitée et unifiée ? Dans la continuité de la théorie sociale, on ne peut quêtre frappé en étudiant le capitalisme de l'ingéniosité et de l'inventivité du capitalisme pour se déployer et perdurer. Marx " est resté à distance des idées qui nous sont devenues familières de diversité, de variété, de pluralité, donc de souplesse et de résilience du capitalisme " (Haber 2015: 540). Une vision anhistorique du capitalisme incite Marx à penser l'essentielle unité du capitalisme ou bien une " erreur téléologique » qui consiste à vouloir soumettre le cours de l'histoire au grand mythe émancipateur du XXe siècle (Bidet 2015). Le contexte des écrits de Marx ainsi que le manque de recul historique sur le capitalisme industriel le justifie cependant.

Sur la base de la caractérisation générale du mode capitaliste, différentes formes de capitalisme se distinguent au cours de l'histoire. La première phase du capitalisme, avec la première révolution industrielle, se caractérise par un entreprenariat bourgeois, de petites entreprises le plus souvent familiales et l'échange des biens sur le marché. Le directeur acquiert son statut et sa légitimité de la propriété, du patrimoine. Il est entrepreneur de risques, calculateur de profit et considéré comme un acteur clé du progrès économique et social. La deuxième phase du capitalisme se situe dans les années 1930 à 1960. Une nouvelle forme de coordination s'impose, outre le marché : l'organisation. C'est la naissance de la grande entreprise, organisée de façon hiérarchique et rationnelle par des principes qui se veulent de nature scientifique, définis par Taylor et appliqués initialement par Ford. Contrairement à la première forme de capitalisme où le marché est le principe de régulation des échanges, selon un principe de régulation spontanée ou invisible (Smith 1991), la grande entreprise de cadres sorganise au contraire selon la " main visible » des managers (Chandler 1989). Cette organisation du travail est caractérisée par une nouvelle forme de pouvoir, la technocratie (Galbraith 1989). La grande entreprise industrielle moderne, entreprise intégrée ou « entreprise multidivisionnaire " (Chandler 1989) devient peu à peu l'institution centrale de nos sociétés. Elle se caractérise par son gigantisme, la concentration spatiale massive des travailleurs et la diversité des activités, à la fois horizontalement et verticalement, au sein d'un même établissement. Dans une troisième phase du capitalisme, au tournant des années 1970-1980, la financiarisation des entreprises permet aux actionnaires d'arriver aux commandes de l'entreprise, imposant une nouvelle intensification du travail au prix de constantes réorganisations et de dispositifs de gestion toujours plus sophistiqués. C'est dans ce contexte et dans celui des critiques libertaires des années 1968 que prend place la transformation managériale des années 
1980-1990. La troisième phase du capitalisme dit « capitalisme réticulaire » permet d'allier les exigences d'autonomisation et de responsabilisation des travailleurs avec un plus grand engagement des salariés, condition sine qua non de l'intensification du travail. Les nouvelles méthodes de management remettent peu à peu en cause les procédés de contrôle et de surveillance pour une moralisation ou responsabilisation du travailleur.

Après avoir analysé le caractère historique du capitalisme, interrogeonsnous à présent sur la spécificité du néocapitalisme à partir des trois caractéristiques fondamentales du système capitaliste: l'idéologie ou le nouvel esprit du capitalisme, les formes d'exploitation d'un nouveau type de capital à accumuler et les nouvelles formes d'aliénation derrière les processus de subjectivation spécifiques.

L'idéologie néocapitaliste ou le nouvel esprit du capitalisme: la forme «réseau»

Si le capitalisme perdure et se réinvente à chaque époque, c'est parce qu'un " esprit » l'accompagne, c'est-à-dire un discours qui permet de le légitimer, de le justifier en tant que système économique (Weber 1964). Les différentes formes du capitalisme ne peuvent pas se penser indépendamment des discours normatifs qui les accompagnent. Ces discours s'articulent autour de trois valeurs : l'autonomie, la sécurité et le bien commun (Boltanski et Chiapello 1999). La transformation du capitalisme tient au primat de certaines de ces valeurs sur les autres. À chaque phase du capitalisme, un « nouvel esprit du capitalisme ", une nouvelle justification se forme en réponse aux critiques formulées à son encontre (Boltanski et Chiapello 1999). Le capitalisme s'adapte et se nourrit de ses critiques, ce qui explique sa longévité. Luc Boltanski et Eve Chiapello soulèvent deux types de critiques adressées au capitalisme : la « critique artiste » et la " critique sociale » (Boltanski et Chiapello 1999). La première se formule en termes d'autonomie et d'authenticité. Elle prend naissance dans le courant contestataire des années 1968, visant prioritairement l'organisation de type bureaucratique. La deuxième vise la dénonciation de l'exploitation et des inégalités sociales et s'est développée plus tardivement, en réponse au néolibéralisme. Dans les années 1970-1980, l'esprit du capitalisme s'est approprié la critique artiste ou critique de l'aliénation, à travers ce que nous pouvons appeler non plus une «biopolitique " comme le pensait Foucault, mais un "biocapitalisme" (Haber 2013 : 235-240). En effet, le capitalisme s'est identifié de plus en plus avec le processus vital qui constitue l'expérience immédiate des sujets humains et conduit à l'effacement des frontières entre le système et la vie, entre le travail et la vie, source de son expansion radicale. Le biocapitalisme signifie alors le déploiement nouveau du capitalisme dans la sphère subjective. Cet esprit du néocapitalisme se trouve dans la littérature managériale (Boltanski et Chiapello 1999). On voit 
apparaitre l'idéal d'« empowerment » ou de prise de pouvoir, d'autonomie et de responsabilisation qui s'accompagne d'une injonction à la disponibilité, à l'engagement personnel (don de soi et de son temps) et au développement de soi.

Pour comprendre l'idéologie du néocapitalisme, revenons à la forme " réseau " qui le caractérise, au regard de la forme " organisation » et de la forme "marché » (Powell 1990). Contrairement à l'organisation hiérarchique (forme paradigmatique du deuxième esprit du capitalisme sans s'être substituée intégralement au marché) qui se caractérise par une certaine stabilité, l'organisation en réseau se caractérise par un environnement en permanente évolution auquel le salarié doit s'adapter pour répondre aux besoins de la structure. D'une part, le réseau relie des points distincts, autonomes, alors que l'organisation fonctionnelle, de type organisme, relie des fonctions interdépendantes. D'autre part, alors que l'organisation hiérarchique suppose un contrôle direct et autoritaire de chacune des fonctions par une instance bien identifiée, le réseau s'organise de façon indirecte et informelle par la surveillance des uns par les autres et l'autocontrôle de chacun qui est la condition pour rester dans le réseau. En effet, puisque la présence dans le réseau n'est pas instituée, durable, établie avec une certaine sécurité pour les acteurs, alors être dans un réseau requiert un effort permanent pour se faire reconnaître comme membre du réseau. Il apparaît alors un positionnement et un jeu d'acteurs renouvelé au sein de la forme réseau. Contrairement à la mobilité permanente, au caractère instable, éphémère et intéressée des relations sur le marché, avec le minimum de cadre et de contrainte possible, le réseau pour fonctionner suppose une certaine stabilité, une inscription dans la durée, malgré les changements permanents en son sein. Cette stabilité est permise par une moralisation des relations qui dépend notamment d'un phénomène de confiance mutuelle (Boltanski, Chiapello 1999 : 536). En effet, la moralisation des relations professionnelles est la condition pour faire converger des individus autonomes et responsables.

\section{Le néocapitalisme et les formes d'exploitation d'un capital immatériel}

L'exploitation est renforcée par la mise au travail de capacités humaines (de relation, disponibilité, souplesse, implication affective, engagement, etc.) que le taylorisme, précisément parce qu'il traitait les hommes comme des machines, ne cherchait pas à atteindre et ne pouvait atteindre. Or cet asservissement des qualités humaines remet en question la séparation, inscrite dans le droit, entre le travail et le travailleur. Ce que le travailleur engage dans la tâche dépend de plus en plus de capacités génériques ou de compétences développées hors de l'entreprise et est par là de moins en moins mesurable en termes d'heures de travail, et cela pour un nombre de plus en plus élevé de salariés (Boltanski et Chiapello 1999, Deuxième partie, IV: 368). 
La théorie de l'exploitation prend une nouvelle forme puisque l'extorsion de la survaleur, la maximisation du profit ne tient plus à l'allongement de la durée $\mathrm{du}$ travail, mais à l'intensification du travail par un ensemble de dispositifs managériaux. Contrairement au taylorisme ou à l'organisation scientifique du travail qui déshumanisait le travailleur pour le rendre performant en décomposant les gestes et en calculant le temps de chaque tâche (Linhart 2015), « les gains de productivité passent aujourd'hui tout autant par un management de la subjectivité que par l'organisation scientifique de l'activité » (Renault 2006). Le néomanagement oscille entre humanisation et nouvelles formes d'exploitation de l'intime. La centralité de l'homme au travail, dans sa dimension subjective est désormais la norme (Linhart 2015). De nouvelles stratégies de mobilisation au travail et de nouvelles attentes vis-à-vis des salariés apparaissent : il ne doit pas seulement effectuer une mission, mais s'engager entièrement dans cette mission. C'est l'invention d'un " contrat psychologique » implicite et subjectif qui vient renforcer l'attachement du travailleur par le seul contrat de travail et par les mécanismes de discipline ou d'autocontrôle. De nouveaux «dispositifs d'enrôlement» viennent compléter les dispositifs de finalité et de procédé antérieurs (Dujarier 2015). Le compromis fordiste devient un échange entre l'investissement personnel dans son travail et la promesse d'une réalisation de soi au travail. Se met en place une mobilisation constante de la subjectivité du travailleur, par des méthodes néomanagériales, par des stages de développement personnel, par des impératifs de développement de compétences. Les compétences comportementales, le savoir-être, et non plus seulement le savoir-faire technique, deviennent des variables de production et de productivité qui impliquent de nouvelles formes d'organisation du travail et de management. Il existe désormais une continuité entre le temps de travail et le temps hors travail puisque ce dernier permet également d'enrichir son "portefeuille de compétences » nécessaire à sa carrière. Dans Surveiller et punir, Foucault situe le déplacement du capitalisme au niveau de l'émergence d'une nouvelle classe dominante dont le pouvoir repose non plus sur le capital comme privilège de la propriété sur le marché, mais à présent sur le savoir comme privilège de la compétence dans l'organisation, sans que l'une des formes de pouvoir ne se substitue à l'autre.

Afin de comprendre ces nouvelles formes d'exploitation, revenons sur le contexte économique global, les nouvelles sources de valeur et l'évolution de la grande entreprise ces trente dernières années avec, d'une part, la mondialisation et la concurrence accrue entre les entreprises multinationales et, d'autre part, l'émergence d'un nouveau marché de produits à haute valeur ajoutée. Face à cette reconfiguration de la demande, l'entreprise doit être capable d'innover (Hatchuel et Segrestin 2012). Le cœur de la valeur ajoutée d'une entreprise réside de moins en moins dans ses volumes de production, mais davantage dans sa capacité à s'adapter à la demande du client, dans sa plus grande flexibilité, 
dans la connaissance qui y est développée. Le rapport entre capital et travail est en train de s'inverser puisque « le moteur essentiel de l'essor d'une économie fondée sur la connaissance se trouve dans la puissance du travail vivant » (Negri et Vercellone 2008). En effet, pour innover, il faut des salariés créatifs et non de simples automates (de plus en plus remplacés par des machines) qui reproduisent à l'identique les mêmes gestes, aux mêmes cadences. C'est par conséquent l'organisation du travail qu'il faut repenser. La créativité du salarié repose sur une plus grande autonomie et une responsabilisation du travailleur libéré du poids de la structure hiérarchique. La conception de la ressource humaine comme source de profit et d'accumulation pour l'entreprise a changé : on passe de la ressource humaine comme main-d'œuvre au potentiel humain comme " cerveau-d'œuvre " dans la société du savoir (Volle 2015). À l'organisation en mode processus, c'est-à-dire la répétition d'un ensemble d'étapes pour parvenir à un même résultat final, vient se substituer l'organisation en mode projet qui suppose le traitement inédit d'une demande circonscrite dans le temps, dans un environnement incertain, en constante réorganisation. Ce mode d'organisation permet l'innovation, la création de nouvelles solutions à valeur ajoutée. L'organisation en mode projet connait elle-même, depuis les années 2000, de nouvelles méthodes dites " agiles » qui remplacent petit à petit les méthodes classiques. Elles reposent sur quatre valeurs : le primat des individus et de leurs interactions par rapport aux processus et aux outils, la prise en compte des bonnes pratiques avant les procédures, la collaboration avec le client plutôt que la contractualisation rigide et enfin l'adaptation permanente au changement. L'entreprise agile se caractérise par une plus grande perméabilité aux changements et une meilleure adaptation pour proposer des produits à haute valeur ajoutée, dans un contexte concurrentiel. Pour cette raison, la grande entreprise ne se caractérise plus aujourd'hui par son degré d'intégration et de concentration en un même lieu des forces productives, mais au contraire par un processus de "désintégration » ou d'extériorisation de ses activités, par le recours à la sous-traitance, à l'intérim notamment (Weinstein 2010). En effet, il faut être capable d'aller chercher la bonne ressource au bon moment.

Le néocapitalisme et les formes d'aliénation ou de rationalisation de la subjectivité

Un nouveau type de subjectivité est mis à l'honneur, celui d'un individu conçu comme "entrepreneur de lui-même» (Foucault 2004), selon une généralisation de la forme entreprise, caractérisé par une attitude concurrentielle et une posture accumulatrice, en rupture avec "l'homme de l'échange " du libéralisme classique (Bissonnette et Cukier 2017). Le vocabulaire gestionnaire ne touche plus seulement les tâches à accomplir, mais vise le travailleur lui-même comme « capital humain » (Schultz 1959) à faire 
fructifier. On passe d'une logique de métier et de qualifications à une logique de compétences transverses (Zarifian 1999). Aux capacités qui conféraient une valeur propre au travail, une capacité à faire, se substitue un "portefeuille de compétences » à acquérir comme valeur d'échange sur le marché du travail. C'est pourquoi les notions de développement personnel et d'employabilité (la somme des compétences accumulées par un salarié) deviennent centrales dans l'entreprise aujourd'hui. On a vu se développer ces trente dernières années des stages de développement personnel ou du coaching. La prise en compte de la personne humaine dans son intégralité, notamment dans sa dimension affective, devient un prétexte pour mieux la gouverner (Brunel 2008). En effet, "l'objectif du coach n'est pas de se mettre à l'écoute des affects mais de "gérer" des émotions » (Le Coz 2015). La subjectivation du travailleur irait dans le sens d'une mainmise plus grande sur les capacités du travailleur, c'est-à-dire d'une plus grande efficacité des forces productives. On peut y voir une extension du champ de la rationalisation aux affects, aux émotions, aux comportements. Autrement dit, il existerait désormais une forme de rationalisation de la vie intérieure, une technicisation de la subjectivité. Il se jouerait au travail une intériorisation des logiques et des exigences de l'organisation : efficacité, productivité et flexibilité.

Le néocapitalisme a pourtant ceci de particulier qu'il caricature la vie plus qu'il ne l'oppresse, il fascine par son dynamisme propre, celui de la vitalité même (Haber 2013). En effet, la culture entrepreneuriale est basée sur l'enthousiasme, l'excitation, l'adhésion, la vitalité, le renouvellement. Elle fait appel à l'intelligence, à la créativité, au génie. La technique et les automatismes ne se produisent plus sans nous et contre nous. La subjectivité se cale sur la puissance expansive du capitalisme plutôt que de la subir. L'instrumentalisation de la subjectivité est rendue possible par deux anthropotechniques du néolibéralisme : le marketing et le management. Par ces anthropotechniques, le capitalisme contemporain se renforce en détruisant toute contestation possible, en se souciant de l'adhésion des individus au système. Le néocapitalisme tient sa force, sa condition de possibilité et de pérennité de la complicité des salariés. Ils participent au déploiement du système capitaliste dans sa logique de productivité et d'accumulation, bien loin d’être uniquement oppressés par un système qui leur serait étranger. Il s'agit davantage d'une connivence, que d'une servitude volontaire puisqu'il se joue la promesse d'une libération et d'une plus grande autonomie du travailleur. Et l'attrait du système devient une condition de sa pérennité.

Après avoir caractérisé la forme spécifique du néocapitalisme, dans l'histoire longue du capitalisme, par son idéologie propre et ses modes d'exploitation et d'aliénation, nous allons interroger à présent la façon dont les nouvelles technologies (notamment les technologies relationnelles de réseau social d'entreprise) s'inscrivent dans les transformations récentes du capitalisme. 


\section{Les réseaux numériques et le capitalisme contemporain}

Le déplacement de l'idéologie du capitalisme en réseau: de la critique artiste à la critique sociale

Les réseaux numériques s'inscrivent dans l'idéologie du fonctionnement en réseau, permettant une plus grande agilité et flexibilité de la force de travail. En effet, les technologies numériques d'information et de communication permettent d'échanger de manière transverse, de partager des bonnes pratiques, en temps réel, là où les processus et les procédures deviennent obsolètes. Mais il semble que les organisations insistent aujourd'hui davantage, dans leur communication, sur l'aspect collaboratif, relationnel, social que sur l'aspect libérateur et flexible des nouveaux modes d'organisation du travail et des outils mis à disposition des salariés. La création de valeur dans l'entreprise contemporaine dépend dorénavant de capacités à créer, à innover qui requièrent un échange d'informations, un travail collaboratif et une intelligence collective. Nous entrons dans un capitalisme cognitif où l'information et la connaissance deviennent le nouveau capital immatériel à accumuler (Hardt et Negri 2000 ; Moulier-Boutang 2007 ; Vercellone 2014) grâce aux relations, aux échanges et aux partages. "L'hypothèse fondamentale sous-tendant cette approche est donc que l'innovation, la connaissance, la capture de la créativité et de l'intelligence sociale distribuée tendent désormais à se présenter comme les principaux vecteurs de création de valeur et d'accumulation du capital » (Sarrouy 2013). Ainsi donc, la production de valeur repose dorénavant en grande part sur le capital de connaissances emmagasiné dans la marchandise. Or le capital immatériel, contrairement au capital matériel, sera d'autant plus important qu'il existe une synergie des forces productives, une synergie des intelligences et non une simple coordination extérieure et addition des forces physiques. La production de valeur devient essentiellement une production sociale. Le paradigme de l'interaction et de la collaboration devient central pour penser la création de valeur au sein d'un capitalisme cognitif. La forme " réseau " devient le modèle d'une organisation collaborative qui suppose une transparence étendue, une horizontalité des relations et un partage de l'information, dans une idéologie communicationnelle. Ainsi, si dans les années 1970-1980, l'esprit du capitalisme a étouffé la critique artiste en pensant l'autonomie et la responsabilisation du travailleur, les exigences de la critique sociale restent quant à elles d'actualité. Il se jouerait aujourd'hui, avec les technologies relationnelles notamment, l'appropriation par le capitalisme d'une nouvelle critique, la critique sociale qui vise à dénoncer l'individualisation des travailleurs, l'affaiblissement du lien social professionnel ou ce que MarieAnne Dujarier appelle des « rapports sociaux sans relation » (Dujarier 2015), en rejoignant la critique des méthodes néomanagériales de sur-humanisation comme individualisation du travailleur (exemplifiées par l'entretien individuel 
annuel) (Linhart 2015). Ce qui nous intéresse à présent est de comprendre ladite "révolution numérique » dans le déplacement qui se joue sur la notion de réseau, non plus uniquement dans le sens d'une plus grande autonomie et mobilité des acteurs, mais bien dans le sens d'une prise en compte de la nécessaire collaboration entre acteurs dans l'entreprise à l'ère du numérique. Et si « dans un monde en réseau, l'importance du capital social et du capital d'information sont corrélés »(Boltanski et Chiapello 1999), c'est bien le réseau informatique qui permet la rencontre et la convergence instantanée des deux sources de valeur du néocapitalisme. Leur complémentarité est à la base du projet de réseau social numérique dans les années 2000, au sein de ce qu'il est courant d'appeler la " deuxième révolution numérique », du « web 2.0 » ou « web social » (O’Reilly 2007). Le réseau social numérique dans les organisations, le réseau social d'entreprise (RSE) est à comprendre sur le modèle des réseaux sociaux grand public à la fin des années 2000, au sein du capitalisme réticulaire et d'un nouveau déplacement dans l'histoire du capitalisme.

Selon la rhétorique managériale, le réseau numérique permet de faciliter la constitution d'un réseau social par l'extension permise des contacts, la rapidité de la constitution d'un réseau et la simplification des codes de communication. Premièrement, le réseau social numérique permet de contacter plus facilement des personnes éloignées géographiquement, mais aussi de statut, de fonction ou de service différent. Il permettrait, au moins dans ses ambitions affichées, une plus grande horizontalité et transversalité dans l'entreprise, ce qui limiterait les phénomènes de reproduction sociale bien présents dans les phases antérieures du capitalisme. Cependant, la constitution de son capital social ou de son réseau de relations sur les outils numériques est fondamentalement inégalitaire (Granjon et Lelong 2006). En effet, pour constituer son propre capital de relations, il faut être à une place convenable, digne de confiance pour les autres membres du réseau, il faut être initié à la recherche de relations stratégiques et détenir certaines compétences communicationnelles. Les nouveaux dispositifs numériques requièrent des compétences numériques qui dépassent la seule maîtrise des outils et désignent davantage une posture, un ensemble de capacités transverses, de nature davantage communicationnelle que technique (Bouillon 2015). Deuxièmement, le réseau numérique permet la rapidité de la constitution d'un réseau. Dans un contexte de changement permanent, les dispositifs numériques sont particulièrement précieux pour permettre l'adaptabilité de chacun. En effet, le réseau numérique simplifie les codes de communication. La publication sur le réseau ne requiert pas de formalisme, de formules de politesses, d'attention à la hiérarchie des destinataires entre destinataires principaux et destinataires en copie, etc. Mais, si les codes de communication se trouvent simplifiés, ils ne disparaissent pas pour autant puisque les réseaux numériques requièrent la maîtrise de nouveaux codes, des outils informatiques, des enjeux de la visibilité notamment. Par exemple, 
l'interaction sur les réseaux en ligne exclut les grands monologues, demande de diversifier les supports de communication en ajoutant des photographies, des vidéos, des émoticons pour exprimer ses affects, etc. Ainsi, il serait faux de dire que le différentiel de langage, de culture, de maîtrise des outils disparaît avec les outils numériques, même s'il s'en trouve parfois simplifié.

De nouvelles logiques d'exploitation dans l'entreprise numérisée: socialisation et invisibilisation de la production

De nouvelles formes d'exploitation dans l'entreprise numérisée reposent sur le modèle économique bâti autour de contenus générés par les collaborateursutilisateurs. Le dispositif de réseau social d'entreprise permet la captation de la valeur produite par les salariés-usagers. L'exploitation se définit alors comme une production de valeur sur internet non immédiatement assignée comme telle (Casilli 2015 : Moulier-Boutang 2007) : « une majorité des contributions bénévoles peut de ce fait être considérée comme du surtravail, un travail producteur de valeur non payé » (Broca 2015). Cette forme d'exploitation dans l'entreprise numérisée peut être comprise sur le modèle du " digital labour » (Fuchs 2014 : Casilli et Cardon 2015 ; Huws 2014). Il désigne le travail des internautes sur les plateformes numériques, à la fois consommateurs et producteurs, générant des profits inédits pour les plateformes. La question posée est celle de la répartition de la valeur induite par ce modèle économique (Scholz 2012). Dans son dialogue avec Dominique Cardon sur le digital labour, Antonio Casilli (2015) dénonce la subsomption du social sous le marchand, contrairement aux représentations naïves, à l'origine d'internet, d'une collaboration volontaire, désintéressée et gratuite constitutive de communs. Si l'approche du digital labour, dans la continuité du courant postopéraïste avec la théorie du capitalisme cognitif et de l'exploitation du travail immatériel (Antonio Negri \& Michael Hardt 2000 ; Yann Moulier-Boutang 2007-; Carlo Vercellone 2014) suppose de distinguer l'emploi, c'est-à-dire les activités exercées dans le cadre d'un contrat de travail, et le travail (labour) comme ensemble des activités créatrices de valeur, il semble que le travail non contractuel envahit également la sphère du salariat. Le digital labour concerne également la grande entreprise capitaliste. Si la production de valeur excède la sphère du travail rémunéré, elle se fait pourtant également au cœur même du système salarial classique puisque les salariés sont invités à s'investir sur les plateformes de réseau social, en plus de leurs missions. Les utilisateurs des plateformes numériques, publiques ou privées, produisent des données, volontairement ou non, avec différents usages que ceux imaginés par le contributeur. Par exemple, une simple réponse à question peut entrer dans une bibliothèque de pratiques ; le renseignement de ses compétences sur sa fiche profil peut permettre le recrutement interne sur projet ou la mobilité interne, etc. 
À partir de l'analyse du travail du consommateur par Marie-Anne Dujarier (2009), nous pouvons distinguer trois types de travail bénévole du salarié. Le premier repose sur la simplification des tâches qui permet la réalisation par n'importe qui de tâches qui excèdent sa fonction. Elles ne donnent pas lieu à évaluation dans le cadre de l'entretien annuel d'évaluation ni donc à rétribution, si ce n'est une reconnaissance tacite de l'investissement personnel du salarié. Dans le cadre des communautés en ligne sur le RSE, cette forme déguisée de travail productif se rencontre sous la forme de la "communautés de service » (Benghozi et al. 2001) sur le modèle des " foires aux questions » ou FAQ pour " frequently asked questions », en anglais (littéralement «questions fréquemment posées »). La plateforme de réseau social d'entreprise permet par exemple une assistance informatique en ligne immédiate et efficace puisque le potentiel de réponse satisfaisante est multiplié par le nombre d'acteurs présents en même temps sur la plateforme. Cela permet de capitaliser sur les savoirs et les pratiques des utilisateurs. Le deuxième type de travail bénévole du salarié, sur le modèle du crowdsourcing, se fonde sur l'ensemble des données, informations, savoirs et pratiques renseignés par la foule des salariés sans répondre à une demande précise et qui constitue un fond commun disponible pour chacun et pour l'entreprise dans son ensemble. A fortiori, toutes les informations renseignées sur son profil, ses compétences, les projets réalisés, etc. et qui peuvent servir à la constitution de groupes projets optimisés, aux mutations internes, à l'évaluation annuelle facilitée par exemple. Le troisième type de travail bénévole du salarié relève d'un " travail d'organisation ", dans la continuité du management participatif et de l'autonomisation du travailleur. En effet, chacun sur le réseau organise le savoir (à l'aide de mots-clés ou " hashtag "), commente la production d'information de ses collègues (à l'aide des fonctionnalités « like » et « commentaire ») donc évalue et juge les comportements des autres sur le réseau.

Avec les réseaux sociaux numériques, nous passons d'une économie de l'échange et de la production à une économie de la pollinisation et de la contribution (Moulier-Boutang 2010). La création de la valeur propre au web est comparable au processus de pollinisation : de la même manière que le calcul de la valeur créée par les abeilles ne peut se résumer à la seule production de miel, mais doit prendre en compte le travail de pollinisation qu'elles effectuent (pour un tiers de la production agricole mondiale), sur le web la valeur est issue de la multiplicité des interactions, des traces et des clics des utilisateurs. Ainsi, les lignes de recoupement, héritées du marxisme, entre production de la plus-value et exploitation se déplacent : « aujourd'hui, la plus-value est en grande partie directement sociale, c'est ce qui est accaparé de la pollinisation générale par le dispositif d'accumulation des pouvoirs » (Moulier-Boutang 2008 : 128). La contribution de chacun devient difficilement évaluable sur un réseau numérique où la création de valeur se veut volontaire et sociale. Les plateformes de RSE posent la question de l'exploitation sous la forme de 
l'intensification du travail et de la charge de travail : faire plus dans le même temps, être polyvalent pour preuve de son implication, pour son évolution de carrière, son employabilité. L'intensification du travail se caractérise alors par deux mécanismes : la socialisation et l'invisibilisation du travail. La socialisation de la production se définit par le décalage entre de nouvelles formes de production socialisée et des cadres professionnels traditionnels individualisés soit un décalage entre une contribution collective et une rétribution (reconnaissance et rémunération) individuelle. L'invisibilisation du travail sur le RSE désigne un travail « en plus ». Les activités sur le RSE font partie non pas de missions obligatoires, prescrites et encadrées, mais au contraire elles sont à l'initiative de l'employé (qui peut tout de même y être contraint) parce que "ludiques », " attractives ", « amusantes ", « fun ", etc., selon la communication d'entreprise.

De nouvelles formes d'aliēnation dans l'entreprise numérisée: rationalisation des compétences relationnelles et stratégie relationnelle

L'aliénation au travail se déplace à présent vers des formes psychosociales nouvelles, non seulement vers de nouveaux processus de subjectivation, mais également vers de nouveaux processus de socialisation. Nous avons vu en quel sens le néocapitalisme s'intéresse non plus seulement aux savoirs et savoir-faire ou compétences techniques, mais également au savoir-être ou compétences comportementales des personnes. A présent, ces compétences comportementales sont développées essentiellement dans un sens relationnel. C'est bien le savoir-être en équipe qui intéresse l'entreprise aujourd'hui. Contrairement à toute la gamme d'outils et de méthodes qui ont été développés depuis les années 1980 pour favoriser l'individualisation des salariés (définition et évaluation du travail, formation, rémunération, évolution de carrière, etc.), on assiste aujourd'hui à la mise en place dans les entreprises d'une stratégie informationnelle, communicationnelle et relationnelle (ateliers de codéveloppement, communautés de pratique, communautés en ligne sur le réseau social d'entreprise, etc.). Les dispositifs numériques jouent un rôle essentiel dans le développement de lêtre-en-relation au travail par le développement de compétences numériques et la " rationalisation des médiations organisationnelles " : "l'enjeu sous-jacent au développement des compétences numériques semble bien se positionner sur le plan de l'optimisation des interactions sociales et des comportements professionnels qui leur sont associés»(Bouillon 2015 : 99). Puisque la productivité des entreprises repose sur la capacité des salariés à interagir, la rationalisation de l'activité et les modes de gestion se portent désormais sur la socialité.

Outre la rationalisation des compétences relationnelles, l'aliénation se porte sur une dépossession du rapport à l'autre. En effet, l'équipe acquiert un rôle de premier plan dans l'organisation en réseau, mais il s'agit de 
liens stratégiques, éphémères, à accumuler. La socialité, dans le sens d'une capitalisation de ses relations devient un enjeu central de reconnaissance individuelle, d'employabilité, de développement de carrière et donc de pouvoir au sein de l'organisation. Le réseau désigne ainsi une multiplication des connexions, mais qui peut se faire de façon purement stratégique et éphémère, non structurante et non constitutive du lien social. En effet, il semble que le capitalisme réticulaire réponde à la volonté d'autonomie tout autant qu'à celle de lien social au travail. Le renforcement des relations professionnelles entrerait en contradiction avec l'idée de mobilité, d'agilité et de flexibilité inhérente à l'organisation en réseau. Il existe un hiatus entre les logiques de don et de contre-don mises en avant et la dimension utilitariste des relations sur le réseau numérique. Par exemple, sur les plateformes relationnelles se joue une " institutionnalisation des principes de don " sous la forme d'une " rationalisation du lien communautaire émancipatoire " (Galibert 2015). Il convient alors de s'interroger sur le « sens émancipateur ou réificateur du rapport « donneur-créancier » / " receveur-débiteur » qui précède le moment du contre-don »(idem). La forme contemporaine de l'aliénation repose donc sur la dimension stratégique et intéressée de la relation à l'autre, entre le développement de capacités relationnelles et la constitution d'un capital social. À ce titre, il semble que le rapport de priorité se soit déplacé parmi les trois formes de capitalisation possibles qui contribuent à la construction et la reproduction des systèmes de différenciation sociale : le capital économique (revenus, propriété des moyens de production, patrimoine), le capital culturel (ressources symboliques, dispositions et qualifications intellectuelles), et le capital social (l'ensemble des relations personnelles que peut totaliser un individu) (Bourdieu 1985). Si le capital social restait secondaire car dépendant du capital économique et culturel, nous pouvons nous demander s'il n'est pas davantage déterminant aujourd'hui pour asseoir sa position et étendre ses possibilités d'accumulation. Les transformations du capitalisme contemporain résident dans la centralité du capital social ou l'accumulation de relations stratégiques permettant d'étendre sa sphère d'influence, soit la position du "faiseur de réseau » (celui qui constitue un réseau relationnel pour son propre intérêt) et non du « mailleur " (celui qui le fait pour le bien commun) (Boltanski et Chiapello 1999). La constitution de son réseau devient un enjeu professionnel stratégique. Non seulement une accumulation est possible dans le domaine de la relation professionnelle, mais il existe une stratégie relationnelle pour se positionner en tant qu'acteur incontournable dans un réseau qui se joue dans les " trous structuraux ", c'est-à-dire les points qui ne sont pas encore reliés entre eux et permettent une "efficacité relationnelle » (le rapport entre le nombre de ses relations non redondantes et le nombre total de ses relations [Burt 1992]).

Le capitalisme contemporain détermine finalement un nouveau rapport à soi. Son identité se construit par l'ensemble de ses relations et interactions 
dans le réseau. Elle devient une "identité relationnelle " (Cardon 2009) dans un double sens. D'une part, le choix des relations est stratégique pour construire son identité numérique. D'autre part, l'individu construit sur les réseaux numériques une identité stratégique pour développer son réseau de relations. Les technologies relationnelles sont un nouveau type de dispositif permettant de construire son identité et sa relation à l'autre : un ensemble d'éléments permettant de faire confiance et de se montrer digne de confiance. En effet, la base de profils disponibles dans le réseau social permet idéalement de sélectionner le bon contact en fonction de sa position dans l'organisation, les informations qu'il est susceptible de détenir, ses compétences affichées (qui relèvent du déclaratif), son réseau de relations et son appartenance à d'autres communautés. Donc la question n'est plus de trancher entre ontologie relationnelle et ontologie substantielle, puisque le primat de l'être-en-relation sur l'être substantiel semble désormais un réquisit de la création de valeur et de l'action, mais bien de déterminer ce qui au sein d'une ontologie relationnelle fait sens : l'individu porteur de relations ou la création de valeur propre à la relation, qui ne saurait être accaparée par tel ou tel ?

Vers un renouveau de la critique sociale: une approche compréhensive de la technologie comme pensée de la transformation sociale

Comment situer la critique aujourd'hui vis-à-vis de la technologie? Quel est le potentiel critique des nouvelles technologies? Les nouvelles technologies peuvent-elles participer à une transformation sociale? Les transformations sociales actuelles ne peuvent être comprises ni selon le modèle techniciste qui magnifie le rôle de la technique ni selon un modèle dualiste qui nie au contraire toute influence de la technique, mais selon le modèle sociotechnique qui permet d'inscrire les technologies numériques dans leur contexte d'appartenance : l'histoire longue du capitalisme, la totalité politique, sociale et économique dans laquelle elles se situent, ainsi que leurs usages (Guéguen 2014). Contrairement à l'idée d'un déterminisme technique ou d'une univocité de la technique, les nouvelles technologies doivent se comprendre dans un contexte de contingence qui laisse place à plusieurs alternatives possibles et à une pensée critique (Feenberg 2004). Selon l'approche constructiviste critique de Feenberg, une critique de la technique, pour proposer une transformation possible, doit révéler les choix, valeurs et intérêts desquels résultent les techniques.

C'est finalement à un travail de déconstruction des dispositifs numériques, de ses pratiques et discours qu'il faut procéder pour poursuivre le travail critique et la transformation sociale. En effet, ce sont dans les usages et discours qui accompagnent la technologie que se jouent les possibilités d'une transformation sociale. Il faut pour cela distinguer la matérialité des outils, ce qu'ils rendent possible, de leurs usages qui peuvent être variés. D'une 
part, la conception des outils n'est pas neutre. D'autre part, les usages sont à définir en se méfiant des "bonnes pratiques " mises en avant par certaines entreprises, cabinets de conseil et centres de formation qui résultent d'une stratégie en amont. La communication sur les nouveaux outils de réseau social dans l'entreprise met en exergue les usages collaboratifs de ces technologies, en mettant de côté l'ensemble des usages stratégiques, égoïstes, utilitaristes qui ne sont pas moins visibles. Le rôle de la critique en matière de nouvelles technologies n'est pas de critiquer tout usage des technologies relationnelles, mais de montrer que leur usage collaboratif est loin d'être exclusif et ainsi de décrypter les usages sous-jacents. Si les TNIC peuvent permettre de développer des savoirs, des savoir-faire et des savoir-être comme bien commun, elles peuvent aussi contribuer à rationnaliser et à capitaliser les interactions dans un sens éminemment stratégique. Elles peuvent aussi permettre de contrôler davantage le travailleur en gardant une trace de ses actions, pensées et relations. Avec le numérique, nous vivons une « crise de transition » (Volle 2015) qui nous impose un choix à prendre. La question n'est pas de savoir si l'on doit poursuivre l'informatisation en marche, mais comment tirer parti des ressources qu'apporte l'informatisation tout en mettant en garde contre ses dangers.

\section{Conclusion}

Pourconclure,le déploiementrécent des nouvelles technologies relationnelles de type réseau social d'entreprise s'inscrit dans l'histoire du capitalisme, dans la continuité du capitalisme réticulaire et en prolonge les potentialités en termes de stratégie d'accumulation d'un capital social et informationnel. Si l'idéologie qui accompagne la forme contemporaine du capitalisme réticulaire propose un ensemble de caractéristiques enthousiasmantes articulées autour de l'idéal collaboratif, dans une perspective de développement personnel par la socialité, il en résulte de nouvelles formes potentielles d'aliénation (dépossession et rationalisation du rapport à l'autre au sein du réseau) et d'exploitation (extorsion de survaleur non rétribuée pour une série de contributions sociales invisibles). L'étude des plateformes de réseau social d'entreprise permet une mise en visibilité des contradictions de l'organisation collaborative : entre ouverture des possibles et poursuite des logiques capitalistes. En effet, le nouvel esprit du capitalisme numérique intègre et anticipe les revendications autour d'une démocratisation des savoirs et des pratiques, de la construction d'un bien commun et d'une centralité du lien social, dans l'univers pourtant traditionnellement hiérarchisé et normé de l'entreprise. 


\section{BIBLIOGRAPHIE}

AUBERT N. et de GAULEJAC V., 1991, Le coût de l'excellence, Paris, Seuil.

Barbrook R. et Cameron A. 1996, "The Californian Ideology ", Science and Culture, $\mathrm{n}^{\circ} 26$, p. 44-72.

BIDET J., 2015, « Foucault avec Marx : pouvoir-capital et pouvoir-savoir », Marx E̋ Foucault, Paris, La Découverte, p. 286-297.

BISSONNETTE J.-F.et CUKIER A.,2017, «Présentation du dossier : néolibéralisme et subjectivité ", Terrains/Théories, n 6. URL : http://teth.revues.org/896

BOLTANSKI L. et CHIAPELLO È., 1999, Le Nouvel Esprit du capitalisme, Paris, Gallimard.

BOUILLON J. L., 2015, " Technologies numériques d'information et de communication et rationalisations organisationnelles : les “compétences numériques" face à la modélisation ", Les Enjeux de l'information et de la communication, n 16, p. 89-103.

BOURDIEU P., 1985, "The Forms of Capital ", in Handbook of Theory and Research for the Sociology of Education, New York, éd. J. G. Richardson, p. 241-258.

BRUNEL V., 2008, Les Managers de l'âme. Le développement personnel en entreprise, nouvelle pratique de pouvoir? Paris, La Découverte.

BURT R., 1992, Structural Holes. The Social Structure of Competition, Cambridge, Harvard University.

CAILLÉ A., 2007, Anthropologie du don, Paris, La Découverte.

CAILLÉ A. et GRÉSY J-E., 2014, La révolution du don. Le management repensé, Paris, Seuil.

CARDON D., 2009, « L'identité comme stratégie relationnelle », Hermès, La Revue, $\mathrm{n}^{\circ}$ 53, p. 61-66.

CASTEL R., 1995, Les métamorphoses de la question sociale, Une chronique du salariat, Paris, Fayard.

CHANDLER A., 1989, La main visible des managers. Une analyse bistorique, Paris, Economica.

DEJOURS C., 1999, Souffance en France, Paris, Seuil.

DUJARIER M-A., 2015, Le management désincarné. Enquête sur les nouveaux cadres du travail, Paris, La Découverte.

FEENBERG A., 2004, Repenser la technique : vers une technique libératrice, Paris, La Découverte/MAUSS.

FLICHY P., 2008, «Technique, usage et représentations », Réseaux, vol. 26, n 148149, p. 147-174.

FOUCAULT M., 1975, Surveiller et Punir, Paris, Gallimard.

FOUCAULT M., 2004, Naissance de la biopolitique, Cours au collège de France (19781979), Paris, Gallimard.

GALBRAITH J. K., 1989, Le nouvel État industriel, Paris, Gallimard.

GRANJON F. et LELONG B., 2006, "Capital social, stratifications et technologies de l'information et de la communication. Une revue des travaux français et anglo-saxons », Réseaux, no 139, p. 147-181. 
GRANJON F., 2012, Reconnaissance et usages d'Internet. Une sociologie critique des pratiques de l'informatique connectée, Paris, Presses des Mines.

GRANOVETTER M., 1973, " The Strength of Weak Ties », American Journal of Sociology, 78, p. 1360-1380.

GUÉGUEN H., 2014, « La critique et le possible : le rôle de la catégorie de possible dans la critique des TNIC », in George, É. \& Granjon, F. (dir.), Critique, sciences sociales et communication, Paris, Mare et Martin.

HABER S., 2008, " Expérience du travail, expérience de l'aliénation », Cités, n 35, p. $45-54$.

HABER S., 2013, Penser le néocapitalisme, Vie, capital et aliénation, Paris, Les prairies ordinaires.

HABER S., 2015, « Diversité et historicité du capitalisme d'après le Livre I du Capital : origines, évolutions, transformations, différenciations ", Les Études philosophiques, n 154 , p. 539-554.

HARDT M. et NEGRI A., 2000, Empire, Paris, Exils.

HARVEY D., 2005, A Brief History of Neoliberalism, Oxford, Oxford University Press. HATCHUEL A. et Segrestin B., 2012, Refonder l'entreprise, Paris, Seuil.

HONNETH A., 2000, La lutte pour la reconnaissance, Paris, Cerf.

LINHART D., 2015, La comédie humaine du travail. De la déshumanisation taylorienne à la sur-humanisation managériale, Paris, Érès.

MARX K., 1993, Le Capital, Paris, PUF.

MARX K., 2007, Les manuscrits économico-philosophiques de 1844, trad. F. Fischbach, Paris, Vrin.

MARX K. 2014, Contribution à la critique de l'économie politique, Paris, Les Éditions sociales.

MAUSS M., 1973, Essai sur le don: Forme et raison de l'échange dans les sociétés archäqques, in Sociologie et Anthropologie, Paris, PUF.

MERCKLÉ P., 2016, La sociologie des réseaux sociaux, Paris, La Découverte.

Moulier Boutang Y., 2007, Le Capitalisme cognitif. La nouvelle grande transformation, Paris, Éditions Amsterdam.

MOULIER BOUTANG Y. (dir.) et al., 2008, « L'entrée dans le capitalisme cognitif », Esprit, $n^{\circ} 11$, p. 123-137.

MOULIER BOUTANG Y., 2010, L'abeille et l'économiste, Paris, Carnets Nord.

NEGRI A. et VERCELLONE C., 2008, « Le rapport capital / travail dans le capitalisme cognitif », Multitudes, $n^{\circ}$ 32, p. 39-50.

PAUGAM S., 2000, Le salarié de la précarité, Paris, PUF.

POWELL W. W., 1990, "Neither market nor hierarchy: Network forms of organization ", Research in organizational behavior, 12, p. 295-336.

RENAULT E., 2006, « Du fordisme au post-fordisme : Dépassement ou retour de l'aliénation? ", Actuel Marx, n³ 39, p. 89-105.

SARROUY O., 2013, «Pistes de réflexion pour une approche communicationnelle critique des organisations dans le contexte du capitalisme cognitif » in Heller, Thomas, 
Huët, Romain, Vidaillet, Bénédicte, Communication et organisation: perspectives critiques, Presses Univ. Septentrion, p. 57-66.

SCHULTZ T., 1959, "Investment in man: an Economist's view », Social Service Review, vol. 33, n², p. 109-117.

SMITH A., 1991, Recherches sur la nature et les causes de la richesse des nations, Paris, Flammarion.

TURNER F.,2006, From Counterculture to Cyberculture : Stewart Brand, the Whole Earth Network, and the Rise of Digital Utopianism, Chicago, University Of Chicago Press.

VERCELLONE C., 2014, «From the Mass-WorkerTo Cognitive Labour : Historical and Theoretical Considerations ", in M. van der Linden et K. H. Roth (éd.), Beyond Marx: theorising the global labour relations of the twenty-first century, Leiden, Brill, $\mathrm{n}^{\circ} 56$.

VOIROL O., 2011, «L'intersubjectivation technique : de l'usage à l'adresse. Pour une théorie critique de la culture numérique », in Denouël J., Granjon F., (dir.), Communiquer à l’ère numérique. Regards croisés sur la sociologie des usages. Paris, Presses des Mines, p. 127154.

VOLLE M., 2015, « Comprendre l'informatisation », in Cabiers Philosophiques, n 141, p. 87-103.

WEBER M., 1964, L'éthique protestante et l'esprit du capitalisme, Paris, Librairie Plon.

WEINSTEIN O., 2010, Pouvoir, finance et connaissance. Les transformations de l'entreprise capitaliste entre $X X^{\mathrm{e}}$ et $X X \mathrm{I}^{\mathrm{e}}$ siècles, Paris, La Découverte.

ZARIFIAN P., 1999, Objectif Compétence. Pour une nouvelle logique, Paris, Liaisons.

Résumé : Cet article vise à comprendre comment ladite "révolution numérique » et, plus précisément, la révolution du web social de ces toutes dernières années s'inscrit dans un contexte de transformations plus large, celles du capitalisme et de la grande entreprise. Avec les technologies relationnelles, le capitalisme contemporain se trouve dans une position paradoxale de réappropriation de la critique sociale sous le paradigme de la collaboration tout en développant de nouvelles formes d'aliénation et d'exploitation du social.

Mots clés : capitalisme, réseau, numérique, technologies relationnelles, exploitation, aliénation.

Abstract : This article aims to understand how the "digital revolution" and, more specifically, the social web revolution of the last few years is part of a broader transformation, that of capitalism and big companies. With relational technologies, contemporary capitalism finds itself in a paradoxical position of reappropriating social criticism under the paradigm of collaboration while developing new forms of alienation and exploitation of the social.

Keywords : capitalism, network, digital, relational technologies, exploitation, alienation. 